\title{
STUDY OF PLACENTAL HISTOPATHOLOGY IN IDIOPATHIC INTRAUTERINE GROWTH RETARDATION- A COMPARATIVE STUDY
}

\author{
Remya Achuthan', Jini L. Valooran², Chandrika C. Velayudhan³, Binesh Balachandran ${ }^{4}$ \\ 1 Junior Resident, Department of Obstetrics and Gynaecology, Government Medical College, Thrissur, Kerala. \\ ${ }^{2}$ Assistant Professor, Department of Pathology, Government Medical College, Thrissur, Kerala. \\ 3 Professor, Department of Obstetrics and Gynaecology, Government Medical College, Thrissur, Kerala. \\ ${ }^{4}$ Senior Resident, Department of Paediatrics, Division of Neonatology, PGIMER, Chandigarh.
}

ABSTRACT
BACKGROUND
Intrauterine growth restriction (IUGR) is one of the most common morbidity encountered by gynaecologists and neonatologists,
and placental pathologies are attributed as an aetiological factor in many cases.
Aim- To seek out the incidence of various placental pathologies in cases of idiopathic IUGR and compare this data with the
placentas of normal weight controls.

\section{MATERIALS AND METHODS}

We included 50 placentas from idiopathic IUGR pregnancies, and 50 placentas from normal foetal weight pregnancies. We included pregnancies complicated by IUGR with no other high risk factors, and excluded those with obvious risk factors. All the placentas were subjected to systematic macroscopic and microscopic analysis.

Statistical Analysis- Statistics methods, using SPSS version 20.

Settings and Design- Analytic study, conducted at Government Medical College, Thrissur, Kerala.

\section{RESULTS}

Baseline demographic characteristics were similar in both groups. Significant placental infarcts were found in 15 of IUGR group and none in control group ( $\mathrm{p}<0.001)$. Syncytial knots occurring $>30 \%$ of the high power fields occurred in 15 of IUGR group and four of the control group ( $\mathrm{p}$ 0.005). Massive perivillous fibrin deposits occurred in 22 (44\%) of the IUGR group compared to 3 (6\%) in the control group ( $\mathrm{p}<0.001)$. The incidence of calcifications, chronic villitis, retroplacental clots and abnormal cord insertion were similar in both the groups.

\section{CONCLUSION}

Our study shows that the placentas of seemingly idiopathic cases of IUGR have a very high incidence of macro and microscopic abnormalities, thus highlighting the probable placental aetiology in these cases.

\section{KEYWORDS}

Histopathology, Intrauterine Growth Retardation, Placenta, Pregnancy.

HOW TO CITE THIS ARTICLE: Achuthan R, Valooran JL, Velayudhan CC, et al. Study of placental histopathology in idiopathic intrauterine growth retardation- A comparative study. J. Evolution Med. Dent. Sci. 2017;6(32):2615-2618, D0I: $10.14260 /$ Jemds/2017/565

\section{BACKGROUND}

Intrauterine growth restriction is a term applied to foetuses that are at or below 10th percentile for their expected birth weight at a given gestational age. IUGR is an important cause of perinatal morbidity and mortality with the incidence in developed countries being 3\%, and in developing countries $15-25 \%$. $^{(1)}$ It is one of the commonly recognised abnormalities of foetal condition and is an important compounding factor in $26 \%$ or more of still births. IUGR babies are at an increased risk of neurodevelopmental deficiencies, congenital malformations, meconium aspiration

Financial or Other, Competing Interest: None.

Submission 20-02-2017, Peer Review 08-04-2017,

Acceptance 14-04-2017, Published 20-04-2017.

Corresponding Author:

Dr. Jini L. Valooran,

Assistant Professor,

Department of Pathology,

Government Medical College,

Thrissur, Mulangunnathu Kavu (PO)

Thrissur (District)-680596, Kerala.

E-mail: jinivalooran77@gmail.com

DOI: $10.14260 /$ jemds $/ 2017 / 565$

(c) (i) $\ominus$ syndrome, hypoglycaemia, hypocalcaemia, respiratory distress syndrome and several other perinatal complications, and has also been attributed to be a risk factor for cardiovascular events and diabetes in adult life.(2-5) For these reasons, accurate prediction, diagnosis and appropriate management of pregnancies complicated with IUGR are important.

Aetiology of IUGR is diverse. It can be due to maternal, foetal and placental factors. The relationship between maternal and foetal circulation in the placenta is crucial for efficient exchanges of oxygen and nutrients, and various placental pathologies are attributed to be associated with IUGR.(6) But systematic studies seeking the association of specific placental changes and IUGR are scanty in the Indian setting. This is a pity considering the fact that about $30 \%$ of babies born at term are small for dates in this country.(1) Although maternal undernutrition and toxaemia of pregnancy are considered to be important causes for this, there are many cases in which definite risk factors are absent and recent literature suggests primary placental involvement in such cases.(6) In this paper, we are trying to seek out the incidence of various placental pathologies in cases of 
idiopathic IUGR and compare this data with the placentas of normal weight controls.

\section{MATERIALS AND METHODS}

This is an analytic study done in the department of Obstetrics \& Gynaecology, Government Medical College, Thrissur in collaboration with the department of pathology during the period from 2011-2012.

We included pregnancies complicated by IUGR with no other high risk factors. Placenta from multiple pregnancies, babies with congenital anomalies, those with proven intrauterine infections detected by TORCH screening and those from pregnancies with maternal complications like hypertension, diabetes mellitus, autoimmune disease, thrombophilias or any other chronic illnesses likely to cause IUGR and placental changes were excluded from the study. IUGR is termed if baby's birth weight is less than $10^{\text {th }}$ centile for their gestational age based on Lubchenco chart. (7)

Placenta of IUGR babies are collected from the delivery area in containers (with formalin as preservative) and sent to the Department of Pathology for histopathological examination.

Systematic gross examination of placenta was performed, which includes examination of the membrane insertion site, insertion of umbilical cord, gross anomalies, visible infarcts, and retroplacental haematomas. Umbilical cord is examined 5 $\mathrm{cm}$ from insertion, number of coils per centimetre of cord was noted, cut section examined for number of vessels and cord diameter measured in 2 perpendicular planes and taking the average. Membranes stripped and placental weight is measured. At least 5 samples were taken from each placenta, 2 from maternal and 3 from foetal surface, from morphologically abnormal looking areas or as randomly from normal placentas and including one from cord and one from membrane roll. A single slide from each block was routinely stained with haematoxylin and eosin (H\&E). Study was started after getting clearance from institutional ethics committee and proper written informed consent was obtained from every participant before enrolment.

According to one previous study conducted in Bhopal, the incidence of placental pathology in the form of PVFD was $17 \%$ in IUGR group and $1.8 \%$ in normal pregnancy. (8) We did not get a similar data from the local population. Assuming the same incidence in the present study population and with a study power of $80 \%$ and alpha error of 0.05 , a total of 50 IUGR and 50 controls were required.

Statistical analysis was done by IBM SPSS statistics version 20. Categorical variables are expressed as percentages between the two groups and were analysed by Chi square test and Fisher's exact test wherever applicable. The continuous data are expressed mean (Standard deviation) for normally distributed data and median (1st 3rd quartile) for skewed variables. The normality of distribution was assessed by using skewness and Kurtosis values, P-P plot, Q-Q plot, histogram plotting and by Kolmogorov-Smirnov test. Normally distributed data were analysed by student $t$ test for independent samples and skewed data were analysed by Mann-Whitney U test.

\section{RESULTS}

The demographic baseline characteristics of both groups were depicted in the Table 1 . The important ultrasonographic (USG) parameters were depicted in Table 2.

Among the multiparous women with IUGR, 57.6\% had their previous pregnancies complicated by IUGR and the association was found statistically significant. Regarding the Doppler findings, majority of selected IUGR cases had abnormal arterial Doppler with elevated umbilical artery S/D ratio. Abnormal venous Doppler was seen only in a single case, may be because before the development of a venous Doppler abnormality, pregnancy might have been terminated. In the present study groups, no neonatal deaths were reported.

\section{Macroscopic Findings}

Although the IUGR placentas were smaller in overall size, nearly all (98\% compared to $96 \%$ in control group) had a thickness of $>2 \mathrm{~cm}$ and was not different from the control group in this respect. Retroplacental clots were observed in seven $(14 \%)$ IUGR placentas compared to $5(10 \%)$ in control group and was statistically not different. Most of the placentas in both groups had the cord insertion in central or eccentric location, with marginal cord insertion occurring in two of the IUGR and four of the control group. 18 (36\%) of the IUGR placentas had a cord diameter of $<1 \mathrm{~cm}$, as compared to only $3(6 \%)$ in control cases. The difference was statistically significant with a $\mathrm{p}$ value of $<0.001$. A reduction in the number of coils $(<1 / 5 \mathrm{~cm})$ occurred in four placentas of IUGR group and 3 of the control group and were comparable to each other. Meconium staining of the placentas was observed in 7 IUGR placentas and 1 control placenta, but the difference didn't attain statistical significance ( $p$ 0.06). Significant placental infarcts (infarcts occurring $>10 \%$ of placenta) (Figure 1) happened in 15 of IUGR group and none in control group ( $\mathrm{p}<0.001)$. The infarcts were uniformly distributed across the central and peripheral regions of the placenta.

\section{Microscopic Findings}

Syncytial knots (Figure 2) occurring $>30 \%$ of the high power fields (hpf) occurred in 15 of IUGR group and four of the control group and the difference was statistically significant (p 0.005). Significant calcifications occurred in 40 of the IUGR placentas and 45 of the control placentas, with $p$ value being 0.2 . None of the placentas in both groups had chorangiosis. Features of chronic villitis were detected in 3 of the IUGR placentas and 1 of the control placenta and the difference was not significant ( $\mathrm{p}$ 0.2). Massive perivillous fibrin deposits ( $>30 \%$ of hpf) (Figure 3 ) occurred in 22 (44\%) of the IUGR group compared to $3(6 \%)$ in the control group, and the difference was highly significant $(\mathrm{p}<0.001)$. 


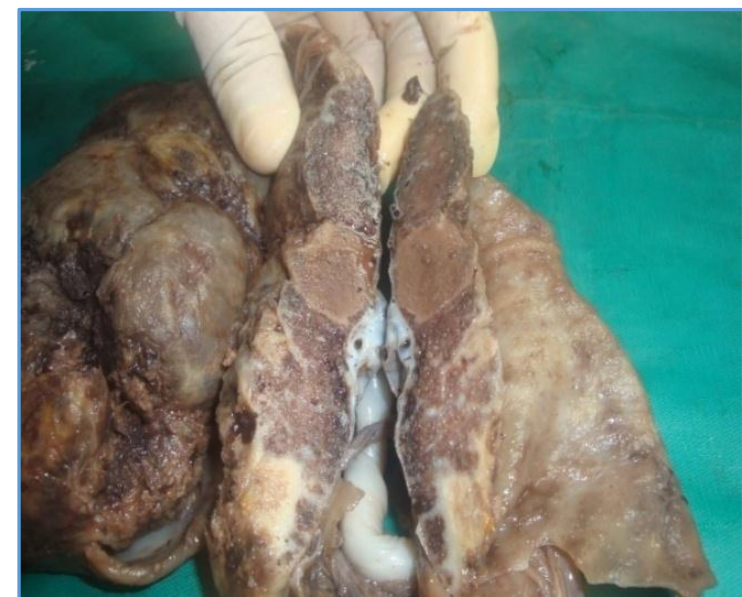

Figure 1. Extensive Placental Infarcts

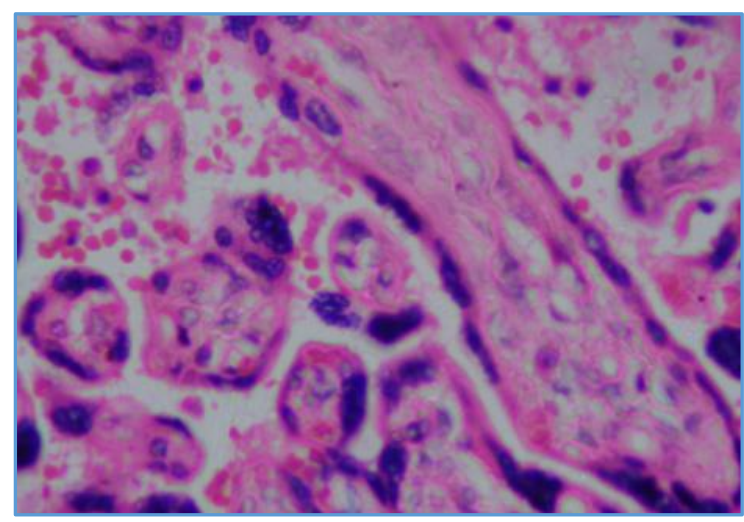

Figure 2. Syncytial Knots

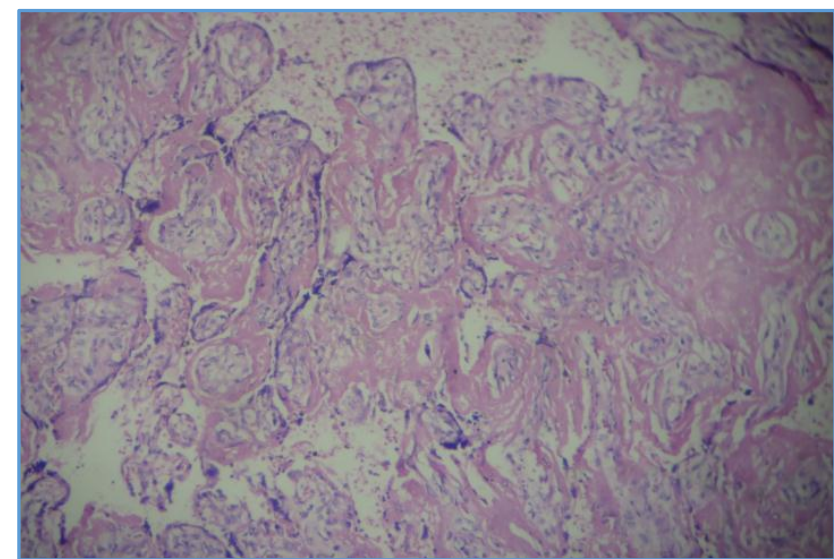

Figure 3. Extensive Perivillous Fibrin Deposits (PVFD)

\begin{tabular}{|c|c|c|c|c|c|}
\hline \multicolumn{2}{|c|}{ Variable } & IUGR Group & Control Group & P value & Mean Difference (CI) \\
\hline \multicolumn{2}{|c|}{ Age* } & $23(21,26)$ & $24(22,28)$ & 0.1 & \\
\hline \multirow{2}{*}{ Period of gestation } & 28-34 wk. † & $50(100)$ & $46(92)$ & \multirow{2}{*}{0.1} & \\
\hline & $35-40$ wk. † & $0(0)$ & $4(8)$ & & \\
\hline \multicolumn{2}{|c|}{ Primigravida $\dagger$} & $20(40)$ & $20(40)$ & 1 & \\
\hline \multicolumn{2}{|c|}{ Maternal smoking $\dagger$} & $0(0)$ & $1(2)$ & 1 & \\
\hline \multicolumn{2}{|c|}{ Maternal BMI $<18.5 \dagger$} & $19(38)$ & $12(24)$ & 0.1 & \\
\hline \multicolumn{2}{|c|}{ Low socioeconomic status $\dagger$} & $38(76)$ & $28(56)$ & 0.03 & \\
\hline \multicolumn{2}{|c|}{ Maternal smoking $\dagger$} & $0(0)$ & $1(2)$ & 1 & \\
\hline \multicolumn{2}{|c|}{ Caesarean delivery $\dagger$} & $11(22)$ & $9(18)$ & 0.6 & \\
\hline \multicolumn{2}{|c|}{ Birth weight $(\mathrm{g}) \neq$} & $2019(375)$ & $2896(359)$ & $<0.001$ & $877(732-1023)$ \\
\hline \multicolumn{2}{|c|}{ Placental weight $(\mathrm{g}) \neq$} & $363(82)$ & $500(84)$ & $<0.001$ & $136(103-169)$ \\
\hline \multicolumn{2}{|c|}{ Number of babies requiring NICU admission +} & $17(34)$ & $2(4)$ & $<0.001$ & \\
\hline \multicolumn{6}{|c|}{ *Median (1st, 3rd quartiles), † Number (\%), $\neq$ Mean (SD) } \\
\hline \multicolumn{6}{|c|}{ Table 1. Demographic and Baseline Variables } \\
\hline
\end{tabular}

\begin{tabular}{|c|c|c|c|}
\hline Variable & $\begin{array}{c}\text { IUGR } \\
\text { Group }\end{array}$ & $\begin{array}{c}\text { Control } \\
\text { Group }\end{array}$ & P value \\
\hline $\begin{array}{c}\text { Abdominal } \\
\text { circumference } \\
<10^{\text {th }} \text { centile }\end{array}$ & $49(98)$ & $1(2)$ & $<0.001$ \\
\hline $\begin{array}{c}\text { Amniotic fluid } \\
\text { index }<5\end{array}$ & $31(62)$ & $1(2)$ & $<0.001$ \\
\hline $\begin{array}{c}\text { Femoral length/ } \\
\text { abdominal } \\
\text { circumference }>23.5\end{array}$ & $24(48)$ & $0(0)$ & $<0.001$ \\
\hline Abnormal Doppler & $28(56)$ & $0(0)$ & $<0.001$ \\
\hline \multicolumn{3}{|c|}{ All variables are expressed as number (percentage) } \\
\hline \multicolumn{3}{|c|}{ Table 2. Ultrasonographic Parameters } \\
\hline
\end{tabular}

\section{DISCUSSION}

IUGR is not a pathological diagnosis but a clinical one with many pathological aetiologies. Understanding the cause of the IUGR in any specific pregnancy is critical for clinical management of future pregnancies and neonatal care. The list of specific causes of IUGR is exhaustive, most aetiologies being rare, but there are some characteristic placental findings. Here lies the importance of histopathological examination of placenta, as the current concept is that most of the so called idiopathic IUGR may infect an underlying placental pathology. In the current study, the average weight of placenta of IUGR was significantly lower than the control group, with the mean difference being $136 \mathrm{~g}$. This was an expected finding, and similar foetal and placental weight difference findings have been reported by many authors. $(9-11)$ 
Significant infarcts involving more than $10 \%$ of placental tissue noticed in $30 \%$ of IUGR cases. Placental infarction has been reported ranging from $0-10 \%$ by different workers $(8,9,12)$ and $100 \%$ by Bhatia et al.(11) Insignificant infarcts involving $<10 \%$ and that too in peripheral location can be seen normally and incidence increases as the gestational age advances. In the present study, in IUGR cases, infarcts were distributed in the periphery as well as in the centre.

Increased number of syncytial knots indicates a degenerative process as a response to local hypoxia. About $30 \%$ of IUGR cases had prominent syncytial knots involving $>30 \% /$ hpf. Nuclear clusters appeared more darkly stained by PAS stain in IUGR group. The terminal villi were prematurely aged as judged by their cytological and histochemical appearance. Ageing of the syncytium is characterised by thinning, basophilia and increased affinity of acid dyes. Although syncytial degeneration does occur under hypoxic results, it is interesting that a concomitant proliferation of cytotrophoblast may occur which might represent an attempt at repair. Similar results were also documented by other authors. $\left.{ }^{8} 8,9,12-13\right)$

Massive perivillous fibrin deposits were seen in $44 \%$ of IUGR placenta and were the most prominent microscopic change that we got from our study. Perivillous fibrin deposits in the intervillous space are a result of thrombosis of maternal blood. The villi embedded in this fibrin are incapable of participating in any transfer activity. Ironically this is seen in placenta with good vascular supply. Greater the blood flow, greater the turbulence and stasis, greater the PVFD. The previous studies showed a lower incidence of PVFD, ranging from $16-36 \%,(8,9,12)$ but many of those studies were based on small sample size.

We didn't find any appreciable difference in the prevalence of calcification nor the presence of findings like chorangiosis, fibrinoid necrosis, and chronic villitis between the 2 groups. Similar results were reported by Kotgirwar et al also.(8)

A number of studies showed pathological changes of the placenta, such as villous infarcts, hypovascularity, fibrosis, and thickening of the basal membrane, obliterative endarteritis, cytotrophoblast proliferation, and syncytiotrophoblasts knotting in patients with IUGR. The presence of two or more of these placental lesions has been shown to be related to perinatal mortality and morbidity.(14) In this study, we also observed that the prevalence of these placental lesions is significantly increased in pregnancies with intrauterine growth restriction. We found that in cases of IUGR with abnormal umbilical artery Doppler velocimetry, the prevalence of villous infarcts, cytotrophoblast proliferation and thickening of the villous trophoblastic basal membrane was significantly higher than those associated to normal umbilical Doppler velocimetry.

\section{CONCLUSION}

Our study showed that the placentas of seemingly idiopathic cases of IUGR had a very high incidence of macro and microscopic abnormalities (Smaller size, infarcts, syncytial knots, massive perivillous fibrin deposits, etc.). Thus, this study highlights the possible aetiological role of placenta in idiopathic IUGR.

\section{REFERENCES}

[1] Saleem T, Sajjad N, Fatima S, et al. Intrauterine growth retardation--small events, big consequences. Italian Journal of Pediatrics 2011;37:41.

[2] Barker DJ, Gluckman PD, Godfrey KM, et al. Fetal nutrition and cardiovascular disease in adult life. Lancet 1993;341(8850):938-41.

[3] Eikenes L, Martinussen MP, Lund LK, et al. Being born small for gestational age reduces white matter integrity in adulthood: a prospective cohort study. Pediatric Research 2012;72(6):649-54.

[4] Lohaugen GC, Ostgard HF, Andreassen S, et al. Small for gestational age and intrauterine growth restriction decreases cognitive function in young adults. The Journal of Pediatrics 2013;163(2):447-53.

[5] Pylipow M, Spector LG, Puumala SE, et al. Early postnatal weight gain, intellectual performance, and body mass index at 7 years of age in term infants with intrauterine growth restriction. The Journal of Pediatrics 2009;154(2):201-6.

[6] Brodsky D, Christou H. Current concepts in intrauterine growth restriction. Journal of Intensive Care Medicine 2004;19(6):307-19.

[7] Lubchenco LO, Hansman C, Dressler $M$, et al. Intrauterine growth as estimated from liveborn birthweight data at 24 to 42 weeks of gestation. Pediatrics 1963;32(5):793-800.

[8] Kotgirwar S, Ambiye M, Athavale S, et al. Study of gross and histological features of placenta in intrauterine growth retardation. J Anat Soc India 2011;60(1):3740.

[9] Bazaz G, Mirchandani JJ, Chitra S. Placenta in intrauterine growth retardation. Journal of Obstetrics and Gynaecology of India 1979;29(4):805-10.

[10] Altshuler G, Russell P, Ermocilla R. The placental pathology of small-for-gestational age infants. American J Obstetrics and Gynecology 1975;121(3):351-9.

[11] Bhatia A, Sharma SD, Jalnawalla SF, et al. A comparative study of placental pathology and fetal outcome. Indian Journal of Pathology \& Microbiology 1981;24(4):277-83.

[12] Mirchandani JJ, Mallik GB, Chitra S. Correlation of fetal outcome with some pathological changes of placenta. J Obstet Gynecol India 1978:1131-9.

[13] Fox H. The morphological basis of placental insufficiency. J Obstet and Gynecol Ind 1975;25(4):441-50.

[14] Viscardi RM, Sun CC. Placental lesion multiplicity: risk factor for IUGR and neonatal cranial ultrasound abnormalities. Early Hum Dev 2001;62(1):1-10. 